\title{
Analgesic roles of peripheral intrinsic met-enkephalin and dynorphin $A$ in long-lasting inflammatory pain induced by complete Freund's adjuvant in rats
}

\author{
YONG-LIANG JIANG, XIAO-FEN HE，YA-FANG SHEN，XIAO-HU YIN, \\ JUN-YING DU, YI LIANG and JIAN-QIAO FANG \\ Department of Neurobiology and Acupuncture Research, The Third Clinical Medical College, \\ Zhejiang Chinese Medical University, Hangzhou, Zhejiang 310053, P.R. China
}

Received April 9, 2014; Accepted November 17, 2014

DOI: $10.3892 /$ etm.2015.2407

\begin{abstract}
Previous studies have focused on strategies for pain relief based on the peripheral opioid system. However, little is known with regard to the profile of the peripheral opioid system in long-lasting inflammatory pain. In the current study, the intrinsic changes of the peripheral opioids were investigated in long-lasting inflammatory pain. A rat model of complete Freund's adjuvant (CFA)-induced inflammatory pain was established. Paw swelling and thermal hyperalgesia (paw withdrawal latency, PWL) were analyzed until day 18 after the CFA injection. The levels of peripheral opioids and their upstream inducers, corticotrophin-releasing factor (CRF) and interleukin (IL)-1 $\beta$, were measured, and validation experiments were performed using opioid receptor antagonists. Long-lasting inflammatory pain was successfully induced in the rats, as shown by the significantly increased paw swelling and decreased PWLs. On day 18 after the CFA injection, the IL-1 $\beta$ levels were significantly elevated, while CRF remained at a normal level in the paw inflammatory tissue. In addition, met-enkephalin (Met-ENK) and dynorphin A (DYN A) levels were significantly increased, while the $\beta$-endorphin level remained normal. Local intraplantar administration of $\delta$ - and $\kappa$-opioid receptor antagonists resulted in more substantial pain, but did not significantly affect the PWLs of the normal control rats. Therefore, the results indicated that the increased levels of local Met-ENK and DYN A in CFA-induced long-lasting inflammatory pain may be involved in peripheral intrinsic analgesia.
\end{abstract}

Correspondence to: Professor Jian-Qiao Fang, Department of Neurobiology and Acupuncture Research, The Third Clinical Medical College, Zhejiang Chinese Medical University, 548 Binwen Road, Hangzhou, Zhejiang 310053, P.R. China

E-mail: fangjianqiao7532@163.com

Key words: peripheral opioid, inflammatory pain, complete Freund's adjuvant, analgesia

\section{Introduction}

The involvement of peripheral opioids in inflammatory pain regulation has been well demonstrated (1-4). Under inflammatory conditions, opioid peptides are released from local immunocytes upon the stimulation of corticotrophin-releasing factor (CRF) and a number of cytokines, including interleukin (IL)-1 $\beta$ (5-7). Among the released opioids are met-enkephalin (Met-Enk) and dynorphin A (DYN A); however, the predominant opioid involved in immune cell-mediated intrinsic antinociception is considered to be $\beta$-endorphin ( $\beta$-END) $(6,8)$. The majority of these findings were obtained from complete Freund's adjuvant (CFA)-induced inflammatory pain with a duration of no more than four days. Considering that long-lasting inflammatory pain is more often encountered in clinical practice than that with a short duration, the changes in peripheral opioids during long-lasting inflammatory pain may provide more valuable insight towards chronic pain control. However, limited data in this area are available at present. In addition, since locally infiltrating immunocyte lineages at different inflammation stages are distinct (9), the profiles of peripheral opioids in long-lasting inflammatory pain may differ from the aforementioned observations.

Thus, the aim of the present study was to systemically assess the profile of peripheral opioids in the later stage of CFA-induced inflammatory pain on day 18 after the CFA injection (10). The levels of $\beta$-END, Met-Enk and DYN A, as well as their upstream inducers, CRF and IL-1 $\beta$, were analyzed, and validation experiments were performed to confirm the intrinsic analgesic effects induced by peripheral opioids in long-lasting inflammatory pain.

\section{Materials and methods}

Animals. Sixteen male Wistar rats (weight, 180-200 g; six-weeks old) were obtained from Shanghai Laboratory Animal Center (Shanghai, China). The rats were housed in a temperature-controlled animal facility $\left(25 \pm 1^{\circ} \mathrm{C}\right)$ under a 12 -h light/dark cycle, with access to food and water ad libitum. The experiments were conducted in accordance with the National Institutes of Health Guide for the Care and Use of Laboratory 
Animals (11). The present study was approved by the Ethics Committee of Zhejiang Chinese Medical Hospital (Hangzhou, China).

Inflammatory pain model. Rats were randomly divided into a normal control group (normal) and CFA-induced inflammatory pain group (CFA), with eight rats per group. Inflammatory pain was induced by an intraplantar injection of $100 \mu \mathrm{l} \mathrm{CFA}$ (Sigma-Aldrich, St. Louis, MO, USA) into the right hind paw. The normal control rats were injected with the same volume of saline. All the rats were sacrificed on day 18 after the injection and samples of the right hind paw inflammatory tissue were obtained. The Wistar rats were used in the first experiment to evaluate the profile change of the peripheral opioids in CFA-induced long-lasting inflammatory pain.

Assessment of inflammation. Inflammation was assessed by the extent of paw swelling. The paw volume was measured in duplicate, using a water displacement plethysmometer (LYS-7A; Shandong Xinhua Medical Instrument Co., Ltd., Zibo, China), prior to the injection of CFA or saline and at the indicated time points following the injection of CFA or saline. Paw swelling was expressed as an increase in paw volume compared with the initial volume.

Evaluation of inflammatory pain. Inflammatory pain was evaluated by assessment of the paw withdrawal latencies (PWLs), as described in our previous study (12). The PWL was measured in triplicate, using a plantar tester (Ugo Basile Srl, Varese, Italy), prior to the CFA or saline injection and at the indicated timepoints following the injection of CFA or saline. Briefly, the rats were placed in a clear plastic chamber and allowed to acclimatize for $30 \mathrm{~min}$. A radiant heat stimulus (high-intensity projector lamp bulb) was positioned under the glass floor directly beneath the right hind paw. When the animal withdrew its hind paw, the heat stimulus was automatically stopped, and the time recorded was the PWL. A 20-sec cut-off was used to prevent tissue injury.

Enzyme-linked immunosorbent assay (ELISA) for CRF and $I L-1 \beta$. Rats were anesthetized with $10 \%(\mathrm{w} / \mathrm{v})$ chloral hydrate at a dose of $3.5 \mathrm{ml} / \mathrm{kg}$ (i.p.) on day 18 after the injection. The right hind paws were immediately removed and rinsed in ice-cold saline. The samples were pulverized in liquid nitrogen, resolved in cell lysis buffer (Beyotime Institute of Biotechnology, Haimen, China) containing protease inhibitor cocktail (10\% v/v; Bio Basic, Inc., Markham, ON, Canada), sonicated on ice $(5 \times 5 \mathrm{sec})$ and stored for $1 \mathrm{~h}$ at $4^{\circ} \mathrm{C}$. The samples were subsequently centrifuged at $13,201 \mathrm{x} \mathrm{g}$ for $30 \mathrm{~min}$ at $4^{\circ} \mathrm{C}$ for protein extraction. The levels of CRF and IL-1 $\beta$ were measured using a rat CRF ELISA kit (Bachem Americas, Inc., Torrance, CA, USA) and rat IL-1 $\beta$ ELISA kit (Enzo Life Sciences, Inc., Farmingdale, NY, USA), according to the manufacturers' instructions, respectively.

Radioimmunoassay (RIA) for $\beta$-END, Mek-ENK and DYN A. Samples for the RIA were collected using the same method as those for ELISA. A RIA was performed to measure the levels of $\beta$-END, Mek-ENK and DYN A in the paw inflammatory tissue at the Department of Neurobiology of the Second Military Medical University of Chinese PLA (Shanghai, China). The levels of $\beta$-END, Mek-ENK and DYN A were measured using a rat ${ }^{125} \mathrm{I} \beta$-END RIA kit, rat ${ }^{125} \mathrm{I}$ Met-ENK RIA kit and rat ${ }^{125} \mathrm{I}$ DYN A RIA kit (Phoenix Biotechnology, Inc., San Antonio, TX, USA), according to the manufacturer's instructions, respectively.

Validation experiment by intraplantar injection of $\delta$-opioid receptor (DOR) antagonist. To determine whether the upregulation of Met-ENK mediated analgesia, the analgesic effect of a DOR antagonist, administered locally on normal control rats and rats with CFA-induced inflammatory pain, was investigated. Thirty male Sprague-Dawley rats (weight, 180-200 g; six-weeks old) were obtained from Shanghai Laboratory Animal Center. These were maintained in the same way as the Wistar rats and were randomly divided into three groups, which included the CFA + saline, CFA + DOR antagonist and normal + DOR antagonist groups $(n=10$ per group). The rat model of inflammatory pain was established by the same method as aforementioned. For the DOR antagonist groups, the rats received an intraplantar injection of $4 \mu \mathrm{g}$ ICI 154,129 (DOR antagonist; Tocris Bioscience, Ellisville, MO, USA) on day 18 following the saline or CFA injection. Rats in the CFA + vehicle group received the same volume of $0.05 \mathrm{ml}$ saline. The PWLs were measured prior to the saline or antagonist injection and $30 \mathrm{~min}$ after the saline or antagonist intraplantar injection.

Validation experiment by intraplantar injection of $\kappa$-opioid receptor (KOR) antagonist. To investigate whether the upregulation of DYN A suppressed chronic inflammatory pain, the effect of a KOR antagonist, administered locally on normal control rats and rats with CFA-induced inflammatory pain, was investigated. Thirty male Sprague-Dawley rats (weight, 180-200 g; six-weeks old) were randomly divided into three groups: $\mathrm{CFA}+$ saline, $\mathrm{CFA}+\mathrm{KOR}$ antagonist and normal + KOR antagonist ( $n=10$ per group). The rat model of inflammatory pain was established by the same method as aforementioned. In the KOR antagonist groups, the rats received an intraplantar injection of $4 \mu \mathrm{g}$ nor-Binaltorphimine dihydrochloride (KOR antagonist; Tocris Bioscience) on day 18 after the CFA or saline injection. Rats in the CFA + vehicle group received the same volume of $0.05 \mathrm{ml}$ saline. The PWLs were measured prior to the saline or antagonist injection and $30 \mathrm{~min}$ after the saline or antagonist intraplantar injection.

Statistical analysis. All data are expressed as the mean \pm standard error of the mean, and were analyzed using the non-paired or paired Student's t-test. All statistical analyses were conducted using SPSS version 18.0 (SPSS Inc., Chicago, IL, USA). $\mathrm{P}<0.05$ was considered to indicate a statistically significant difference.

\section{Results}

CFA successfully induces chronic inflammatory pain. Rats that were administered an intraplantar injection of CFA developed chronic inflammatory pain in the right hind paw, as demonstrated by the significantly increased paw swelling $(\mathrm{P}<0.001$; Fig. 1A) and reduced PWL $(\mathrm{P}<0.001$; Fig. 1B) when compared with the rats in the normal group. 

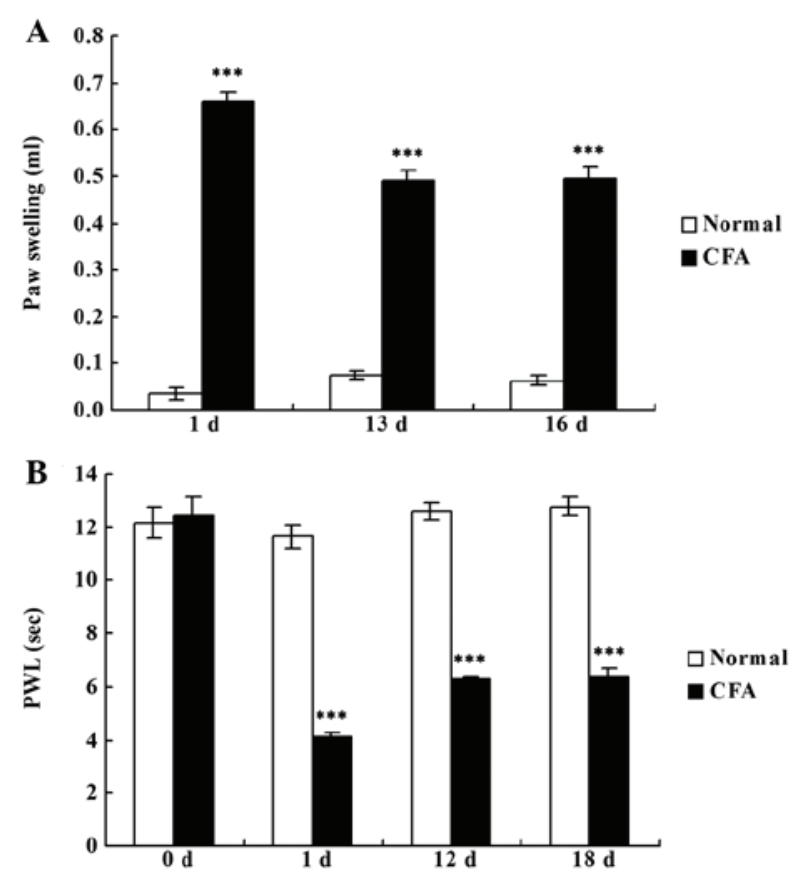

Figure 1. Chronic inflammatory pain was successfully induced by a CFA injection into the right hind paw. (A) Paw swelling was expressed as an increase in paw volume compared with the initial volume. (B) PWL was measured using a plantar tester to evaluate inflammatory pain. Data are expressed as the mean \pm standard error of the mean $(n=8) .{ }^{* * *} \mathrm{P}<0.001$, vs. normal group. CFA, complete Freund's adjuvant; PWL, paw withdrawal latency.
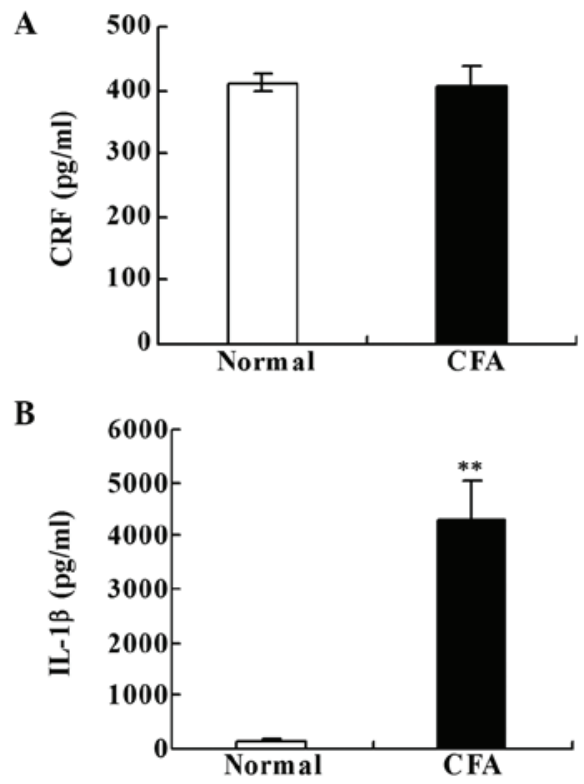

Figure 2. Levels of (A) CRF and (B) IL-1 $\beta$ in the inflammatory tissue of the right hind paw on day 18 after the CFA injection. Data are expressed as the mean \pm standard error of the mean $(n=8) .{ }^{* *} \mathrm{P}<0.01$, vs. normal group. CFA, complete Freund's adjuvant; IL, interleukin; CRF, corticotrophin-releasing factor.

$C R F$ and IL-1 $\beta$ levels in the paw inflammatory tissue. No statistically significant difference in the CRF level was identified between the two groups (Fig. 2A). However, the levels of IL-1 $\beta$ were significantly increased in the CFA group when

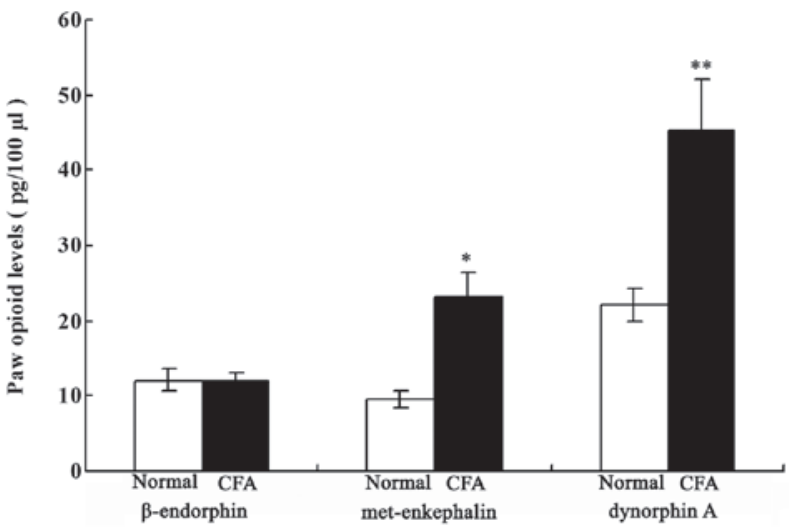

Figure 3. Levels of opioids in the inflammatory tissue of the right hind paw on day 18 after the CFA injection. Data are expressed as the mean \pm standard error of the mean $(n=8)$. ${ }^{*} \mathrm{P}<0.05$ and ${ }^{* *} \mathrm{P}<0.01$, vs. normal group. CFA, complete Freund's adjuvant.

compared with the normal group on day 18 after the CFA injection $(\mathrm{P}<0.001$; Fig. 2B).

Local opioid levels in the paw inflammatory tissue. As shown in Fig. 3, the levels of Met-ENK and DYN A were significantly increased in the CFA group when compared with those in the normal group on day 18 after the CFA injection $(\mathrm{P}<0.05$ and $\mathrm{P}<0.01$, respectively). However, no statistically significant difference in the $\beta$-END level was observed between the two groups.

Effect of the intraplantar injection of the DOR antagonist on chronic inflammatory pain. To investigate whether the upregulation of Met-ENK suppressed chronic inflammatory pain, the effect of ICI 154,129 (DOR antagonist) local administration was examined. Prior to the saline or ICI 154,129 injection, no statistically significant difference in the PWLs of the right hind paws was observed between the CFA + saline and CFA + DOR antagonist groups on day 18. The local ICI 154,129 injection significantly decreased the PWLs of the CFA-injected rats when compared with the CFA + saline group $(\mathrm{P}<0.01$; Fig. 4A). As shown in Fig. 4B, compared with the PWL prior to injection on day 18, local administration of ICI 154,129 significantly decreased the PWLs of the CFA-injected rats (P<0.01; Fig. 4B), but failed to significantly reduce the PWLs of the normal rats.

Effect of the intraplantar injection of the KOR antagonist on chronic inflammatory pain. To determine whether the upregulation of DYN A played a role in intrinsic analgesia, the effect of local administration of nor-Binaltorphimine dihydrochloride (KOR antagonist) was investigated. Prior to the saline or nor-Binaltorphimine dihydrochloride injection, no statistically significant difference in the PWLs of the right hind paws was observed between the CFA + saline and CFA + KOR antagonist groups on day 18. However, the local nor-Binaltorphimine dihydrochloride injection significantly decreased the PWLs of the CFA-injected rats when compared with the CFA + saline group $(\mathrm{P}<0.01$; Fig. 5A). As shown in Fig. 5B, when compared with the PWLs prior to injection on day 18 , local administration of nor-Binaltorphimine dihydrochloride significantly decreased the PWLs of the CFA-injected rats ( $\mathrm{P}<0.01$; Fig. 5B), but failed to significantly reduce the PWLs of the normal rats. 
A

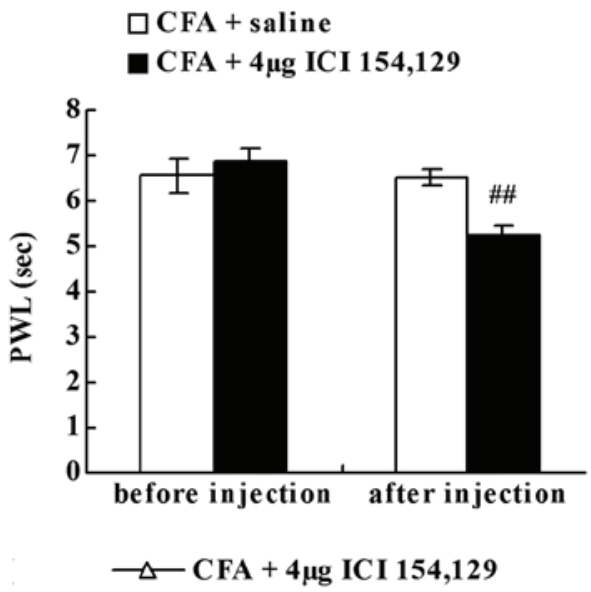

B

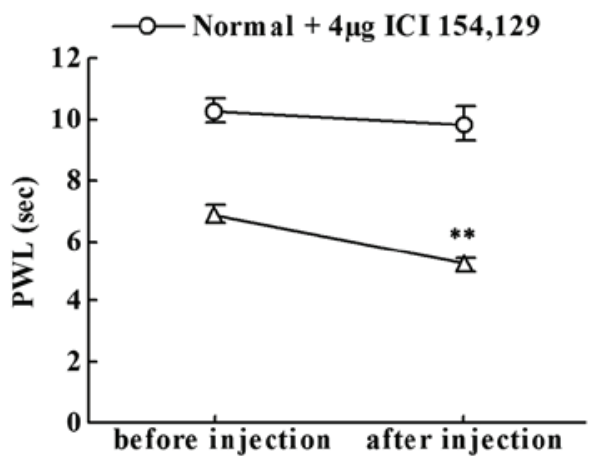

Figure 4. PWL was measured on day 18 after the CFA injection using a plantar tester to evaluate inflammatory pain. (A) Effect of ICI 154,129 (DOR antagonist) and saline administration on the PWLs of CFA-injected rats. (B) Effect of ICI 154,129 (DOR antagonist) on the PWLs of normal and CFA-injected rats. Data are expressed as the mean \pm standard error of the mean $(\mathrm{n}=10) .{ }^{\# \#} \mathrm{P}<0.01$, vs. CFA + saline group; ${ }^{* *} \mathrm{P}<0.01$, vs. CFA $+4 \mu \mathrm{g}$ DOR antagonist group before injection. CFA, complete Freund's adjuvant; PWL, paw withdrawal latency; DOR, $\delta$-opioid receptor.

\section{Discussion}

Peripheral opioids are attracting increasing attention for the development of peripherally restricted strategies for pain relief. However, limited data exist with regard to their profiles in long-lasting inflammatory pain. The present study, for the first time, demonstrated the involvement of peripheral Met-Enk and DYN A in intrinsic analgesia in a rat model of CFA-induced long-lasting inflammatory pain.

Inflammation can trigger the migration of opioid-containing immunocytes to the inflammatory tissue, causing the release of opioid peptides $(13,14)$. Unlike peripheral intrinsic analgesia, which is primarily mediated by $\beta$-END in the early stage of CFA-induced inflammatory pain (on day 4 after CFA injection) $(6,15,16), \beta$-END was shown to remain at an almost normal level in the later stages of inflammatory pain (on day 18 after CFA injection). However, the levels of Met-Enk and DYN A were significantly increased in the paw inflammatory tissue. The different profiles of the peripheral opioids in the early and later stages of CFA-induced inflammatory pain may be the result of the distinct characteristics of the locally infiltrating immunocytes (9). The most commonly referred to stimulators of the peripheral opioids released from immunocytes in inflammatory sites are CRF, IL-1 $\beta$ and other
$\mathbf{A}$
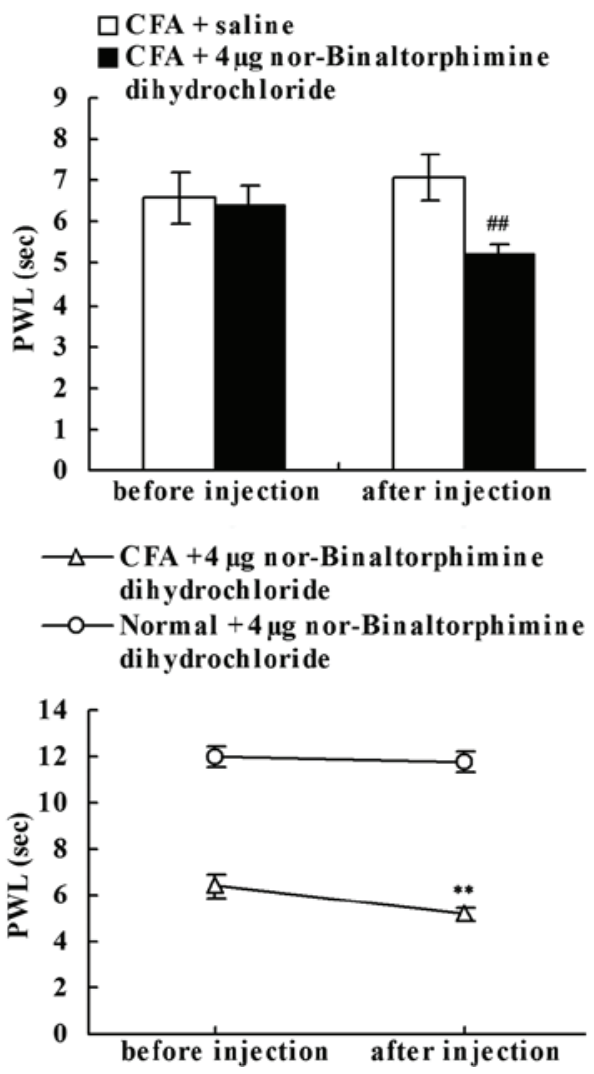

Figure 5. PWL was measured on day 18 after the CFA injection using a plantar tester to evaluate inflammatory pain. (A) Effect of nor-Binaltorphimine dihydrochloride (KOR antagonist) and saline administration on the PWLs of CFA-injected rats. (B) Effect of nor-Binaltorphimine dihydrochloride on the PWLs of normal and CFA-injected rats. Data are expressed as the mean \pm standard error of the mean $(\mathrm{n}=10) .{ }^{\# \#} \mathrm{P}<0.01$, vs. CFA + saline group. ${ }^{* *} \mathrm{P}<0.01$, vs. CFA $+4 \mu \mathrm{g}$ KOR antagonist group before injection. CFA, complete Freund's adjuvant; PWL, paw withdrawal latency; к-opioid receptor.

inflammatory cytokines $(6,17-19)$. A normal CRF level and a significantly elevated IL-1 $\beta$ level were observed in the present study, indicating that the increased levels of peripheral opioids in long-lasting inflammatory pain may be more closely associated with inflammatory intrinsic cytokines, such as IL-1 $\beta$, but not local CRF. CRF levels have been reported to be elevated and play an important role in immune cell-mediated intrinsic antinociception under stress conditions, such as a cold water swim (20).

To testify the hypotheses that upregulation of Met-Enk and DYN A may mediate intrinsic analgesia to prevent further injury or more serious pain $(4,21)$, the effects of the local administration of DOR and KOR antagonists on inflammatory pain were investigated. Locally delivered DOR and KOR antagonists resulted in a more substantial pain to the CFA-injected rats. However, the antagonists did not significantly affect the pain thresholds of the normal rats, indicating that the upregulation of Met-Enk and DYN A contribute to intrinsic analgesia in long-lasting inflammatory pain. These observations are in accordance with a previous study, which demonstrated that locally administered naloxone (opioid receptor antagonist) can exacerbate pain under inflammatory conditions (4). Peripheral intrinsic analgesia mediated by Met-ENK and DYN A may be regarded as the body's negative feedback of inflammation, 
a subsequent protective mechanism to prevent further pain under long-lasting inflammatory conditions.

In conclusion, the present study demonstrated that Met-ENK and DYN A, but not $\beta$-END levels, were increased in long-lasting inflammatory pain, unlike that observed for early-stage of CFA-induced pain where $\beta$-END is the predominant elevated peripheral opioid. In addition, upregulation of Met-ENK and DYN A were shown to contribute to peripheral intrinsic analgesia. The results provide valuable data for developing peripherally restricted strategies for pain relief under long-lasting inflammatory conditions.

\section{Acknowledgements}

This study was supported by grants from the National Natural Science Foundation of China (nos. 81072855 and 81303039), the Zhejiang Provincial Natural Science Foundation of China (nos. Z2100979 and LY12H27015) and the Key Subject of State Administration of Traditional Chinese Medicine of China (Acupuncture and Moxibustion).

\section{References}

1. Tanaka N, Sakahashi H, Sato E, Hirose K and Ishii S: The efficacy of intra-articular analgesia after total knee arthroplasty in patients with rheumatoid arthritis and in patients with osteoarthritis. J Arthroplasty 16: 306-311, 2001.

2. Kalso E, Smith L, McQuay HJ and Andrew Moore R: No pain, no gain: clinical excellence and scientific rigour - lessons learned from IA morphine. Pain 98: 269-275, 2002.

3. Cunha TM, Roman-Campos D, Lotufo CM, et al: Morphine peripheral analgesia depends on activation of the PI3Kgamma/AKT/nNOS/NO/KATP signaling pathway. Proc Natl Acad Sci USA 107: 4442-4447, 2010.

4. Stein C, Hassan AH, Lehrberger K, Giefing J and Yassouridis A: Local analgesic effect of endogenous opioid peptides. Lancet 342 321-324, 1993.

5. Mousa SA, Bopaiah CP, Stein C and Schäfer M: Involvement of corticotropin-releasing hormone receptor subtypes 1 and 2 in peripheral opioid-mediated inhibition of inflammatory pain. Pain 106: 297-307, 2003.

6. Schäfer M, Carter L and Stein C: Interleukin 1 beta and corticotropin-releasing factor inhibit pain by releasing opioids from immune cells in inflamed tissue. Proc Natl Acad Sci USA 91: 4219-4223, 1994.
7. Mousa SA, Shakibaei M, Sitte N, Schäfer M and Stein C: Subcellular pathways of beta-endorphin synthesis, processing, and release from immunocytes in inflammatory pain. Endocrinology 145: 1331-1341, 2004.

8. Stein C, Schäfer M and Hassan AH: Peripheral opioid receptors. Ann Med 27: 219-221, 1995.

9. RittnerHL,Brack A,MachelskaH,etal:Opioidpeptide-expressing leukocytes: identification, recruitment, and simultaneously increasing inhibition of inflammatory pain. Anesthesiology 95: 500-508, 2001.

10. da Silva MA, Bersani-Amado CA, Ishii-Iwamoto EL, Bracht L and Caparroz-Assef SM: Protective effects of indomethacin and cyclophosphamide but not of infliximab on liver metabolic changes caused by adjuvant-induced arthritis. Inflammation 34: 519-530, 2011.

11. National Research Council (US) Committee for the Update of the Guide for the Care and Use of Laboratory Animals: Guide for the Care and Use of Laboratory Animals, 8th edition. Washington (DC): National Academies Press (US), 2011.

12. Ren WK, Yin J, Zhu XP, et al: Glutamine on intestinal inflammation: A mechanistic perspective. Eur J Inflamm 11: 315-326, 2013.

13. Rittner HL, Machelska $\mathrm{H}$ and Stein C: Leukocytes in the regulation of pain and analgesia. J Leukoc Biol 78: 1215-1222, 2005.

14. Mousa SA: Morphological correlates of immune-mediated peripheral opioid analgesia. Adv Exp Med Biol 521: 77-87, 2003.

15. Mousa SA, Zhang Q, Sitte N, Ji R and Stein C: beta-Endorphin-containing memory-cells and mu-opioid receptors undergo transport to peripheral inflamed tissue. J Neuroimmunol 115: 71-78, 2001.

16. Machelska H, Schopohl JK, Mousa SA, et al: Different mechanisms of intrinsic pain inhibition in early and late inflammation. J Neuroimmunol 141: 30-39, 2003.

17. Cabot PJ,CarterL,SchäferMandSteinC: Methionine-enkephalinand Dynorphin A-release from immune cells and control of inflammatory pain. Pain 93: 207-212, 2001.

18. Binder W, Mousa SA, Sitte N, Kaiser M, Stein C and Schäfer M: Sympathetic activation triggers endogenous opioid release and analgesia within peripheral inflamed tissue. Eur J Neurosci 20: 92-100, 2004.

19. Puehler W, Rittner HL, Mousa SA, et al: Interleukin-1 beta contributes to the upregulation of kappa opioid receptor mRNA in dorsal root ganglia in response to peripheral inflammation. Neuroscience 141: 989-998, 2006.

20. Schafer M, Mousa SA, Zhang Q, Carter L and Stein C: Expression of corticotropin-releasing factor in inflamed tissue is required for intrinsic peripheral opioid analgesia. Proc Natl Acad Sci USA 93: 6096-6100, 1996.

21. Sehgal N, Smith HS and Manchikanti L: Peripherally acting opioids and clinical implications for pain control. Pain Physician 14: 249-258, 2011. 